\title{
Fundamental Processes of Photo induced Charge Transfer in Quantum Dot-Based PV Systems
}

\author{
Isa Amos P* \\ Department of Chemistry, Moddibo Adama University of Technology Yola, Nigeria
}

Submission: May 15, 2017 ; Published: June 07, 2017

*Corresponding author: Pigweh Isa Amos, Department of Chemistry, Moddibo Adama University of Technology Yola, Nigeria, Tel: +234-07036759959; Email: Amospigweh@gmail.com

Abstract

In a quest to explore the field of renewable energy sources, Semiconductor Quantum dots has received several nods as light harvesters, emitters and as potential markers in imaging owing to their opto-electronic properties which are size dependent. One of the fundamental but little known aspect of Quantum dots' solar photo conversion is the dynamics of charge transfer. This paper discusses the fundamental processes of charge transfer i.e. Electron injection in Quantum dots to metal oxides composites, Charge trapping in Quantum dots, and finally, Exciton vs Energy Transfer.

\section{Introduction}

Semiconductor Nano crystals commonly referred to as Quantum dots has gained applications as potential markers in imaging, as emitters in light emitting diodes, and as light harvesters in photovoltaic systems. This progress is largely due to the dots' opto-electronic properties which are size dependent and to the fact that they are quantum confined systems. Progress made in wet-chemistry for the synthesis of colloidal quantum dots has stimulated research in their fundamental properties and applications. Of all the applications mentioned, the photovoltaic aspect has received a considerable attention because it has offered the prospect of breaking the Shockley-Quiesser thermodynamic limit of single junction solar cells by using Multiple Exciton Generation [1] or Hot Electron Transfer [2] to improve the efficiency of quantum dots solar cells and hence, posing as world-leading renewable energy source.

On the other hand, the study on the photo induced electron transfer is one of the most fundamental topics in the interdisciplinary research field in physical sciences. This is a process of the photo excitation of donor (D) or acceptor (A) and the subsequent charge separation to produce the chargeseparated state $(\mathrm{D} \bullet++A \bullet-)$ (equation 1 and 2). Depending on the molecule excited by light, photo induced electron transfer can be classified into two types

1. The electron transfer from the excited electron donor

$\left(D^{*}\right)$ to the acceptor $(A)$ and
2. The hole transfer from the donor (D) to the excited acceptor $\left(A^{*}\right)$.

Electron transfer occurs via Lowest Unoccupied Molecular Orbital, while whole transfer occurs via Highest Occupied Molecular Orbital. Finally, charge recombination occurs to reproduce the ground state when the charge-separated state is not converted into electronic or chemical potential (equation 3).

$$
\begin{aligned}
& \mathrm{D}^{*}+\mathrm{A} \rightarrow \mathrm{D} \bullet++\mathrm{A} \bullet- \\
& \mathrm{D}+\mathrm{A}^{*} \rightarrow \mathrm{D} \bullet++\mathrm{A} \bullet- \\
& \mathrm{D} \bullet++\mathrm{A} \bullet-\rightarrow \mathrm{D}+\mathrm{A}
\end{aligned}
$$

Natural photosynthesis is an important example of Photo induced electron transfer and serves as the best example of solar conversion in which Light induced charge separation is achieved through judicious spatial arrangement of the pigments and elements of the electron transport chain in the thylakoids membrane. Co-operative interaction between these components allows the electron transfer to proceed in a vectorial fashion. progress in solar photo conversion are therefore aimed at mimicking this natural process by fabricating crystal structures capable of harvesting and converting sunlight into useful and storable forms, chiefly via Photo induced electron transfer process.

Here, basic processes of photo induced electron/hole transfer in quantum dots-based solar systems are discussed 


\section{Recent Advances in Petrochemical Science}

under the following guidelines; Electron injection in Quantum dots to metal oxides composites, Charge trapping in Quantum dots, and Exciton vs Energy Transfer.

\section{Electron Injection in Quantum Dots to Metal Oxides}

In most solar cell architectures based on Quantum dots as light harvesters, anodic charge transport is carried out by a wide band gap oxide semiconductor [3]. Electrons are injected from Quantum dots to the oxide, generating the photocurrent. This is why one of the most important issues for improving device efficiency is to understand and control the fast Exciton dissociation at the Quantum dot/oxide interface, and charge injection into the anodic material, depending on the relative alignment of conduction and valence-band edges of the core and shell. The rate of electron injection can be affected by; the size of the quantum dots, this is because the size directly affects the driving force of the injection, the core-shell structure since the increase in shell thickness slows down the injection, and the mode of quantum dots-metal oxides attachment.

Most of the studies on electron injection focus on situations where excited electron relaxes into the lowest excited levels in quantum dots and are consequently injected into the metal oxide, however, the electrons can also be injected before the complete relaxation. This process is known as hot electron injection. The hot electron injection enables harvesting of a part of electron's excess energy, which can potentially increase efficiency of a solar cell via the hot carrier extraction through a narrow energy band above the lowest energy levels [2] and utilization of different metal oxides materials in a system similar to multi-junction solar cells [4]. Despite the competing cases of fast electron injection and carrier relaxation, the most effective way to ensure efficient hot carrier harvesting is by careful selection of quantum dots-metal oxide linker since electron relaxation rate in quantum dots-metal oxides can be quite different from the quantum dots itself.

\section{Charge Trapping in Quantum Dots}

One of the factors limiting the efficiency of solutionprocessed colloidal Quantum dot solar cells is the inefficient charge extraction from the active layer of the device. It was shown that the poor charge transport properties of Quantum dot films originates from the high concentration of defect states provided by the Quantum dot surface states $[5,6]$ Such defect states act as trapping centers for the photo generated charges, decrease their charge mobility, enhance recombination, and thereby set a limit to the cell thickness and light-absorption efficiency [7]. Majority of the trapped charge carriers mentioned were reported to be holes. For this reason, the control over the concentration and depth of surface traps has become a major tool for improving the photo conversion efficiency of Quantum dot devices. Such control is usually achieved by the management of ligands surrounding the Quantum dots and atomic-level passivation of their surfaces via the use of core-shell structures, and by directed Exciton energy transfer between quantum dots [5,7-10].

The dynamics of charge generation, trapping and recombination in Quantum dot thin films occurs on multiple timescales, being particularly rich in the first few nanoseconds after the excitation, as demonstrated by a large number of time-resolved spectroscopic studies that probed the carrier dynamics with near- and mid-IR light pulses. These studies make use of the fact that the electronic excitation of Quantum dots leads to the appearance of new absorption bands in the near and mid-IR spectral regions $[6,7,11]$. The origin of this excitation-induced absorption is still debated, as it can be associated with mobile as well as trapped charge carriers or, most likely, represents a combination of responses from both sub-ensembles showing slightly different spectral characters and oscillator strengths.

\section{Exciton Vs Energy Transfer}

Photo-generated electron-hole pairs behaves as excitons in colloidal Quantum dots when there exist a large binding energy [12], the motion of such excitons between Quantum dots occurs via Forster Resonant Energy Transfer (FRET) is essential for the function of optical devices with densely packed Quantum dot films such as solar cells and light emitters [13-15]. In such quantum dot films, low exaction energy enables a rapid dissociation of excitons. As a result, charge transfer between Quantum dots is more efficient than energy transfer. Energy transfer only occurs in case of relatively large Quantum spacing.

\section{Conclusion}

Quantum dots offer a great platform for improved solar energy harvesting achievable through photo induced electron injection which is very fast and efficient, while hole injection are hampered by charge trapping which limits the efficiency of the cell. However, this limiting efficiency can be improved by passivation of the whole traps via the use of core-shell structures. But this is still subject to further studies as future research may seek to go beyond our current knowledge of passivation as a way forward.

\section{References}

1. Klimov V (2007) Spectral and Dynamical Properties of Multi excitons in Semiconductor Nano crystals. Annu Rev Phys Chem 58: 635-673.

2. Ross RT (1982) Efficiency of Hot-Carrier Solar Energy Converters. J Appl Phys 53: 3813.

3. Ruhle S, Shalom M, Zaban A (2010) Quantum dots Sensitized Solar cells. Chem Phys Chem 11(11): 2290-2304.

4. Tvrdy K, Frantsuzov PA, Kamat PV (2011) Photo induced Electron Transfer from Semiconductor Quantum Dots to Metal Oxide Nanoparticles. Proc Natl Acad Sci USA, 108(1): 29-34.

5. Ip AH, Thon SM, Hoogland S, Voznyy O, Zhitomir sky D, et al. (2012) Hybrid Passivated Colloidal Quantum Dot Solids. Nat Nanotechnol 7(9): 577-582. 
6. Tang J, Kemp KW, Hoogland S, Jeong KS, Liu H, et al. (2011) Colloidalquantum-dot photovoltaic using atomic-ligand passivation. Nat Mater 10(10): 765-771.

7. Jeong KS, Tang J, Liu H, Kim J, Schaefer AW, et al. (2012) Enhanced Mobility-Lifetime Products in PbS Colloidal Quantum Dot Photovoltaic. ACS Nano 6(1): 89-99.

8. Liu H, Zhitomirsky D, Hoogland S, Tang J, Kramer IJ, et al. (2012) Systematic Optimization of Quantum Junction Colloidal Quantum Dot Solar Cells. Appl Phys Lett 101: 151112-151113.

9. Malicki M, Knowles KE, Weiss EA (2013) Gating of Hole Transfer from Photo excited PbS Quantum Dots to A minoferrocene by the Ligand Shell of the Dots. Chem Commun (Cambridge, UK) 49: 4400-4402.

10. Tisdale WA, Williams KJ, Timp BA, Norris DJ, Aydil ES, et al. (2010) Hot-Electron Transfer from Semiconductor Nanocrystals. Science. 328(5985): 1543-1547.
11. De Geyter B, Houtepen AJ, Carrillo S, Geiregat P, Gao, Y, et al. (2012) Broadband and Picosecond Intraband Absorption in Lead-Based Colloidal Quantum Dots. ACS Nano 6(7): 6067-6074.

12. Meulenberg R, Lee J, Wolcott A (2009) Determination of the Exciton Binding Energy in CdSe Quantum Dots. ACS Nano 3(2): 325-330.

13. Santra PK, Kamat PV (2013) Tandem-Layered Quantum Dot Solar Cells: Tuning the Photovoltaic Response with Luminescent Ternary Cadmium Chalcogenides. J Am Chem Soc 135(2): 877-885.

14. Zheng K, Zídek K, Abdellah M, Torbjörnsson M, Chábera P, et al. (2012) Fast Monolayer Adsorption and Slow Energy Transfer in CdSe Quantum Dot Sensitized ZnO Nanowires. J Phys Chem A 117: 5919-5925.

15. Choi S, Jin H, Bang J, Kim S (2012) Layer-by-Layer Quantum Dot Assemblies for the Enhanced Energy Transfers and Their Applications toward Efficient Solar Cells. J Phys Chem Lett 3(23): 3442-3447.

\section{Your next submission with Juniper Publishers will reach you the below assets}

- Quality Editorial service

- Swift Peer Review

- Reprints availability

- E-prints Service

- Manuscript Podcast for convenient understanding

- Global attainment for your research

- Manuscript accessibility in different formats

( Pdf, E-pub, Full Text, Audio)

- Unceasing customer service

Track the below URL for one-step submission https://juniperpublishers.com/online-submission.php 